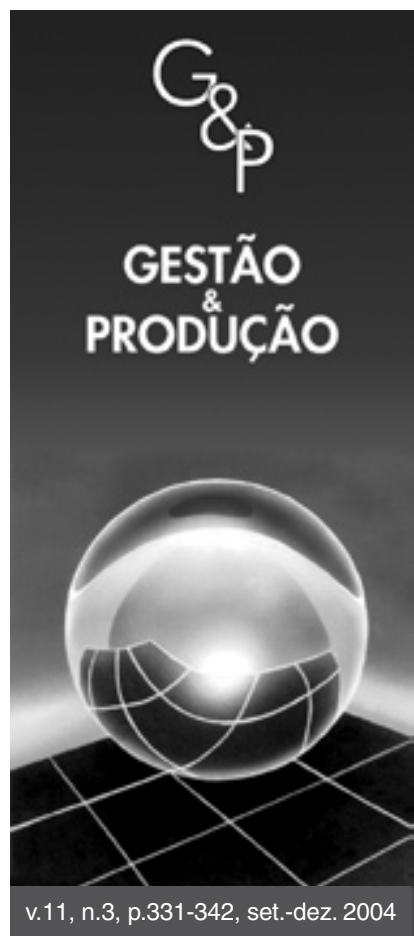

\title{
MÉTODO PARA ANÁLISE DE BENEFÍCIOS EM CADEIAS DE SUPRIMENTO: UM ESTUDO DE CASO
}

\author{
Fernando Cezar Leandro Scramim \\ Faculdade de Engenharia, Engenharia de Produção, \\ Fundação Santo André \\ Av. Príncipe de Gales, 821, CEP 09060-650, Santo André, SP, \\ e-mail: fernandosc@fsa.br \\ Mário Otávio Batalha \\ Departamento de Engenharia de Produção, \\ Universidade Federal de São Carlos, \\ C.P. 676, CEP 13565-905, São Carlos, SP, \\ e-mail:dmob@power.ufscar.br
}

Resumo

Recebido em 07/6/2004

Aceito em 22/11/2004

O objetivo deste trabalho é apresentar um método para realizar análises quantitativas de desempenho de configurações operacionais alternativas, em cadeias de suprimentos empresariais. Este método propõe integrar, em um único framework de análise, os conceitos advindos da gestão da cadeia de suprimentos e de sistemas de custeios gerenciais. O trabalho propõe a operacionalização do método por meio da utilização dos conhecimentos advindos da abordagem conhecida como system dynamics (SD). Para tanto, foi realizado um estudo de caso junto a uma Cooperativa de Laticínios do interior do Estado de São Paulo, no qual modelos de simulação foram construídos, baseados na análise prévia dos elementos do sistema. Deste modo, tornou-se possível examinar as relações de causalidade por meio da construção de cenários alternativos e controle sistemático das variáveis pesquisadas. Do exame dos cenários alternativos e do desempenho do sistema frente aos "distúrbios" aos quais as variáveis são submetidas, natureza do processo de simulação, pôde-se ganhar um maior aprendizado sobre o sistema em estudo.

Palavras-chave: Gestão de cadeias de suprimento, pensamento sistêmico, modelagem computacional.

\section{Introdução}

O enfoque sistêmico está direcionando cada vez mais os estudos relacionados aos problemas afetos ao agronegócio. Na perspectiva sistêmica, a competitividade empresarial exige eficiência interna e interorganizacional. Nesse sentido, é preciso associar competitividade à organização interna eficiente e aos sistemas de comunicação e coordenação de atividades inter-firmas numa cadeia de produção agroindustrial.

O conjunto de idéias ligadas à noção de cadeia produtiva vem se mostrando muito útil nos últimos anos, para pesquisadores e decisores públicos e privados. Entretanto, estas mesmas idéias vêm se mostrando menos eficientes em apontar às empresas ferramentas gerenciais que permitam operacionalizar ações conjuntas, que aumentem o nível de coordenação e de eficiência de uma cadeia agroindustrial (Batalha e Silva, 1999). Entre estas ferramentas gerenciais destacam-se os sistemas de gestão de custos e ferramentas de otimização logísticas. Estes conceitos devem ser expandidos além das fronteiras da firma, para atingir as fronteiras do sistema representado pela cadeia produtiva. Na direção do enfoque gerencial e operacional dos agentes econômicos pertencentes às cadeias produtivas, surge, na literatura recente sobre gestão empresarial, o conceito de gestão da cadeia de suprimentos (do inglês, Supply Chain Management - SCM).

Entretanto, uma questão importante, que se apresenta na literatura sobre gestão de cadeias de suprimento, é a dificuldade de identificação dos ganhos e benefícios mútuos aos agentes econômicos gerados (ou não!), pela aplicação destes conceitos. Nesse sentido, este trabalho propõe a utilização da abordagem sistêmica do system dynamics $(S D)$. Esta abordagem sistêmica pode auxiliar 
no processo de tomada de decisão.

Este trabalho tem por objetivo propor um método para realizar análises quantitativas de desempenho de configurações operacionais alternativas, em cadeias de suprimentos empresarias. Para mostrar a aplicação do método, foi realizada uma aplicação deste junto a uma Cooperativa de Laticínios, no qual um modelo de simulação foi construído, baseado na análise prévia dos elementos do sistema.

Deste modo, apresenta-se, no próximo item, uma revisão teórica sobre a temática de gestão de cadeias de suprimento e as questões advindas da literatura específica. No item 3, faz-se uma breve apresentação da abordagem de $\boldsymbol{S} \boldsymbol{D}$ e seus principais elementos de modelagem. No item 4, apresenta-se a aplicação do método no estudo de caso de uma rede de produtores rurais e a respectiva usina processadora (laticínio). Por fim, algumas considerações finais são estabelecidas em função dos resultados alcançados.

\section{Gestão da cadeia de suprimentos}

Uma cadeia de suprimento deve congregar diversas organizações ou agentes econômicos e institucionais, desde os fornecedores de matérias-primas, passando pelas indústrias de transformação e pelos distribuidores e varejistas, para o atendimento das necessidades dos consumidores finais. Segundo Cooper et al. (1997), o conceito de supply chain management vai além de um novo nome para o conceito de logística. Esses autores utilizam a definição de SCM desenvolvida pelo International Center for Competitive Excellence (1994): “Gestão da Cadeia de Suprimento é a integração dos processos de negócio, desde o usuário (cliente) final até o fornecedor original, gerando produtos, serviços e informações que agregam valor para o consumidor".

Com uma abordagem mais quantitativa e voltada para questões de desempenho econômico de uma cadeia de suprimento, Zimmer (2002) ressalta que, diferentemente da logística tradicional, SCM envolve a coordenação de empresas gerencialmente independentes que buscam maximizar seus lucros individuais. Embora a performance global de uma cadeia de suprimento dependa da performance conjunta das empresas envolvidas, as metas operacionais individuais podem ser conflitantes e resultar em ineficiência para a cadeia como um todo.

Gilbert e Ballou (1999) vão além destes conceitos e advogam que a melhoria da coordenação, em uma cadeia de suprimento, envolve a tentativa de um agente de mudar o comportamento de pelo menos um agente, por meio da oferta de algum tipo de incentivo. Na mesma direção, Zimmer (2002) destaca que embora o custo global de uma cadeia de suprimento seja minimizado, pode ocorrer uma situação na qual o custo de um agente seja reduzi- do, enquanto o custo de outro aumente. Claramente esses autores destacam a questão da melhoria da performance operacional, por meio da redução de custos dos agentes, e do sistema como um todo, como crucial para ganho de competitividade.

De acordo com Gilbert e Ballou (1999), embora haja consenso em torno da existência de benefícios advindos da coordenação nas cadeias de suprimento, há muito que ser feito em termos da identificação e quantificação destes benefícios específicos. Ballou et al. (2000) destacam que o desafio está nas situações, quando os benefícios da cooperação nas cadeias de suprimento não são facilmente identificáveis e quantificáveis, e os mecanismos, para alcançar tais benefícios, também não são bem estabelecidos. Esses autores ressaltam que as ferramentas e técnicas gerenciais tradicionais não são apropriadas para a gestão inter-organizacional de cadeias de suprimento, e que são necessárias novas sistemáticas de gestão de custos (novos sistemas de medição de custos), entre outras.

Novas sistemáticas de apuração de custos, a que se referem os autores, devem ser capazes de definir e medir custos e outros dados relevantes entre os vários elos, para a análise de oportunidades de redução de custos e/ou ganhos de produtividade conjunta, em uma cadeia de suprimento. Os sistemas atuais de gestão de custos são desenvolvidos individualmente para as empresas, e incorporam pouco ou quase nada dos dados de outros agentes da cadeia de suprimento. Fica evidente que um sistema de gestão de custos, integrando as diversas empresas pertencentes ao escopo principal de uma cadeia de suprimento, seria bastante adequado. Este sistema deveria relatar os custos de estoques, de transporte, de produção (custos operacionais), bem como informar as esperadas e desejáveis reduções nos desperdícios do sistema como um todo (ganhos de produtividade), advindos das ações de cooperação inter-organizacionais.

Nesse sentido, questões relativas à estruturação do espaço analítico (a cadeia de suprimento) tornam-se tarefas vitais. Uma cadeia de suprimento como espaço analítico deve congregar elementos operacionais e estratégicos, que possam atuar como fonte de dados, para as análises gerenciais das intervenções à cadeia de suprimento. Esses elementos são relacionados com a definição do número de participantes, com a construção de um direcionamento estratégico conjunto entre as empresas participantes da cadeia, com um estudo das características operacionais e administrativas destes, das potenciais atividades inter-organizacionais que visem a cooperação e dos mecanismos que a assegure, entre outros.

Tentando avançar nessa direção, este trabalho propõe a utilização da abordagem de $\boldsymbol{S} \boldsymbol{D}$ como uma ferramenta gerencial de apoio à tomada de decisão em (re)projetos de cadeias de suprimento, visando avaliar conjuntamente os aspectos econômicos e operacionais do sistema. No 
próximo item, apresenta-se o método de $S D$ e seus elementos básicos de modelagem.

\section{A abordagem de System Dynamics - SD}

Diversos modelos em $S D$ foram desenvolvidos para estudar sistemas econômicos e sociais, durante a última década. Na literatura, podem ser encontrados trabalhos na área como o de Buongiorno (1996), o qual aplicou o $S D$ junto com a econometria para projetar mudanças de capacidade na indústria florestal, em diferentes partes do mundo. Também na indústria de florestas plantadas, para a produção de lápis de madeira no Estado de São Paulo. Yonenaga (2001) desenvolveu uma modelagem em SD para a tomada de decisões econômicas, para corte de florestas de diferentes idades e de conseqüentes rendimentos operacionais, para a indústria processadora. Saito (2000) desenvolveu uma modelagem em $S D$ para identificar pontos de alavancagem na cadeia de suprimento da canade-açúcar brasileira. Smith e Van Ackere (2002) adaptaram em uma modelagem em $S D$ um modelo econômico tradicional de oferta e demanda, no sistema nacional de saúde da Inglaterra.

Sonka e Cloutier (1998) aplicaram a modelagem em $S D$ para explorar o papel das informações de feedback no gerenciamento estratégico dos estoques e fluxos físicos e financeiros, na interface das operações de produção/comercialização de carne de porco, dentro da cadeia suinoculturista dos EUA. Concebida com dados fornecidos pelos participantes do sistema, o modelo proposto da cadeia de valor, formada por produtores rurais (granjas) e empresas processadoras, buscou capturar as interações físicas, econômicas e de desempenho das atividades desenvolvidas ao longo do tempo.

Fisher et al. (2000) utilizaram a modelagem em $S D$ para estudar a adoção e a difusão da tecnologia de mapeamento e monitoramento do campo, por produtores de soja nos EUA. O modelo visou explicitar as relações causais que influenciam a adoção da tecnologia e sua respectiva difusão. Esses autores destacam que os gestores nos negócios agroindustriais estão enfrentando um ambiente dinâmico, complexo e incerto, no qual eles têm que tomar decisões. Além disso, eles destacam também que os fatores que afetam os resultados de suas decisões mudam ao longo do tempo, sendo os resultados impossíveis de serem avaliados na hora que as decisões são tomadas. Avaliar as consequiências das diferentes opções de decisão pode melhorar a performance dos gestores. De acordo com os autores, a modelagem em $S D$ é um tipo de ferramenta gerencial que pode ajudar nesse processo.

Essas abordagens de simulação em $S D$ apresentadas visam permitir que os agentes pertencentes às cadeias de suprimento do agronegócio ganhem um melhor entendimento acerca dos fatores causais (sistêmicos) que influenciam o processo de tomada de decisão. Assim, a modelagem computacional em $S D$ vem ganhando espaço nos meios acadêmico e empresarial como ferramenta de aprendizagem e de apoio à tomada de decisão.

A estrutura do $S D$ é formalizada por meio de modelos de simulação, os quais consistem essencialmente de dois componentes: a rede de estoques e fluxos e a rede de informações. Matematicamente, um modelo de simulação em $S D$ busca representar o mundo como um conjunto de equações diferenciais. A predição do estado do sistema em um dado ponto no futuro é obtida por integração, começando do estado (situação) inicial (Buongiorno, 1996). A abordagem busca modelar explicitamente cada relação causal e traçar o comportamento resultante do sistema ao longo do tempo (Smith e Van Ackere, 2002).

Com o rápido avanço das tecnologias de software é possível, sem muitas dificuldades, construir modelos em $S D$ e testar uma variedade de especificações alternativas. Para representar a estrutura completa do sistema, utilizam-se alguns tipos de ícones e definem-se as conexões entre eles. Conectando-se os ícones, são geradas as equações diferenciais. Além disso, o programa computacional executa todas as integrações, e as principais operações matemáticas, que precisam ser construídas pelo modelador, são adição, subtração, multiplicação e divisão. Cabe destacar que existe a possibilidade de utilização de funções mais avançadas, como as operações de delays (variáveis em atraso), matrizes, testes condicionais, de acordo com a complexidade dos modelos que se quer construir. Para cada ícone no modelo, deve haver uma equação, definindo-o e relacionando-o com pelo menos um outro ícone do modelo (Cover, 1996).

Para a modelagem computacional em $S D$, podem ser utilizados alguns programas comerciais disponíveis no mercado, tais como os pacotes Stella ${ }^{\circledR}$, PowerSim ${ }^{\circledR}$, Vensim ${ }^{\circledR}$ e ithink ${ }^{\circledR}$. Neste trabalho, utilizou-se o software Stella ${ }^{\circledR} 5.0$ e, portanto, os detalhamentos dos procedimentos serão relativos a este pacote computacional.

Os elementos básicos de um modelo computacional em $S D$ são basicamente quatro: estoques, fluxos, conversores e conectores. A Figura 1 apresenta os símbolos utilizados para representar os elementos de um modelo em $S D$. Os estoques são representados por retângulos. Operacionalmente, estoques funcionam como acumuladores. Entretanto, não é necessário que este "estoque" seja apenas resultado de uma acumulação física de bens materiais. Esses "estoques" podem representar, por exemplo, a acumulação de conhecimento que uma organização experimenta num tempo específico.

Estoque e fluxo são agrupados em um modelo juntos, como, por exemplo, o conhecimento e o aprendizado, o tamanho de uma população e a taxa de crescimento, entre outros. Assim, estoques e fluxos são inseparáveis e necessários, para gerar a mudança ao longo do tempo (di- 


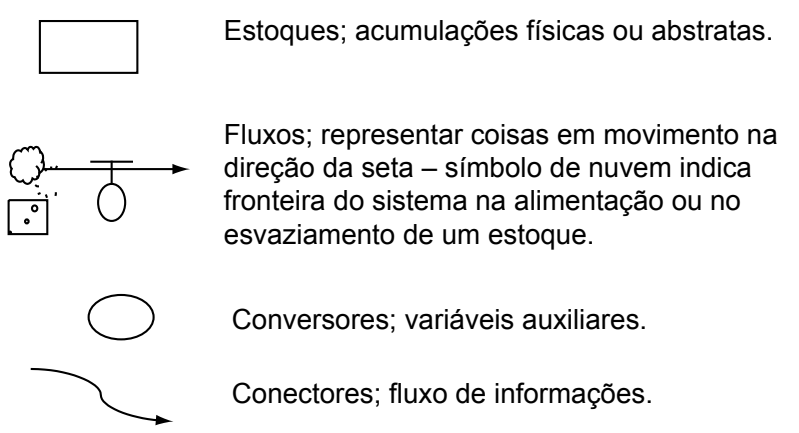

Figura 1. Elementos básicos de modelagem em System Dynamics para o pacote Stella ${ }^{\circledR}$ 5.0 (Stella e Stella Research, 1997).

nâmica). Os fluxos são representados na modelagem por um tubo com uma válvula, um regulador de fluxo e uma ou duas setas indicando o sentido do fluxo. Fluxos conservativos drenam um estoque enquanto alimentam outro. Fluxos não conservativos usam o símbolo de nuvem que indica a fronteira do sistema: uma nuvem na fonte de alimentação de um estoque do sistema indica que, para o propósito do modelo, é considerado como existente um suprimento "ilimitado" daquele item; e uma nuvem na saída de um estoque do sistema indica que uma capacidade "ilimitada" é considerada como existente para absorver aquele fluxo.

Dois outros elementos importantes de um modelo em $S D$ são os conversores (variáveis auxiliares) e os conectores. Conversores são representados por circunferências e podem representar tanto informação quanto quantidades (valores de variáveis). Diferentemente dos estoques, os conversores não acumulam. O valor para um conversor é recalculado em cada "rodada" que os cálculos são realizados (em cada unidade de tempo que o modelo simula o comportamento das variáveis). Os conectores representam o fluxo de informações (setas de informação), mostrando as relações de influência entre os elementos do modelo. Eles representam as entradas e saídas de dados, para os elementos, e podem ligar estoques a conversores, estoques a fluxos reguladores, fluxos a fluxos, fluxos a conversores e conversores a outros conversores (Stella e Stella Research, 1997).

\section{Método de avaliação de cadeia de su- primentos: um estudo de caso}

Em modelagem, a idéia é representar uma pequena parte da realidade. Quando se modela um problema, deseja-se incluir no modelo uma representação da realidade tanto quanto necessário, para gerar um perfeito entendimento do comportamento do problema (Cover, 1996). Uma vez tendo-se um modelo que representa o que está atualmente acontecendo no sistema, tem-se uma ferra- menta para explorar várias mudanças neste.

Realizou-se, inicialmente, uma delimitação da cadeia de suprimentos que represente uma rede de produtores rurais e a respectiva usina de beneficiamento no interior do Estado de São Paulo (Cooperativa de Laticínios), definindo o modelo de referência como sendo composto por uma rede de captação e beneficiamento de leite. Assim, a usina processadora da cadeia de suprimento aqui representada deve ser definida, no modelo de referência, com uma dada capacidade produtiva e com uma gama de produtos beneficiados. Essa cadeia de suprimento deve ser composta também por um grupo de produtores rurais, definidos em número e em volume de produção individual.

O processo de modelagem iniciou-se pela definição do perfil operacional dos produtores rurais, a serem representados no modelo, e seus respectivos fluxos de fornecimento de leite para a Cooperativa. Buscou-se representar a rede de produtores rurais agrupada em quatro categorias, para simplificar o entendimento do processo de modelagem. Estabeleceram-se duas categorias para produtores de leite tipo $\mathrm{C}$ e duas para os produtores de tipo B. A primeira classe de produtores de leite $\mathrm{C}$ refere-se aos de menor porte (produção diária inferior a 150 litros), com 112 unidades rurais. Outras 26 propriedades, com produção diária média acima de 150 litros, compõem a segunda classe de produtores de leite $\mathrm{C}$. Os produtores de leite B foram classificados também em dois grupos: os de pequeno e os de grande porte, sendo que o primeiro grupo representava apenas $14 \%$ do total de leite B captado.

Um processo de levantamento de dados, via entrevistas com questionários semi-estruturados com 23 produtores rurais e com a participação de funcionários da Cooperativa, foi realizado de tal forma a representar todo o universo, e por meio de um processo de amostragem nãoprobabilístico. Esse tipo de amostra baseia-se em julgamento pessoal e deve ser utilizada quando características específicas devem ser encontradas na amostra. Este é o caso do presente estudo, no qual se desejava encontrar, na amostra, representantes de propriedades rurais de diversas capacidades produtivas.

Objetivou-se também levantar o perfil operacional da rede de produtores, assim como levantar dados de custos da operação rural. A planilha de cálculo foi montada com base na lógica do Custeio Direto ou Variável.

A estrutura do modelo (composta pela rede de variáveis auxiliares, estoques e fluxos e a rede de informações) é apresentada na Figura 2. Foram definidos quatro "estoques" no modelo, representando a quantidade de leite recebida pelas usinas do sistema, durante o período considerado (mês) por cada grupo de produtores rurais (os quais não estiveram necessariamente em "estoque" e sim, foram processados e comercializados durante $o$ mês). Assim, fica definida a unidade básica de tempo que 


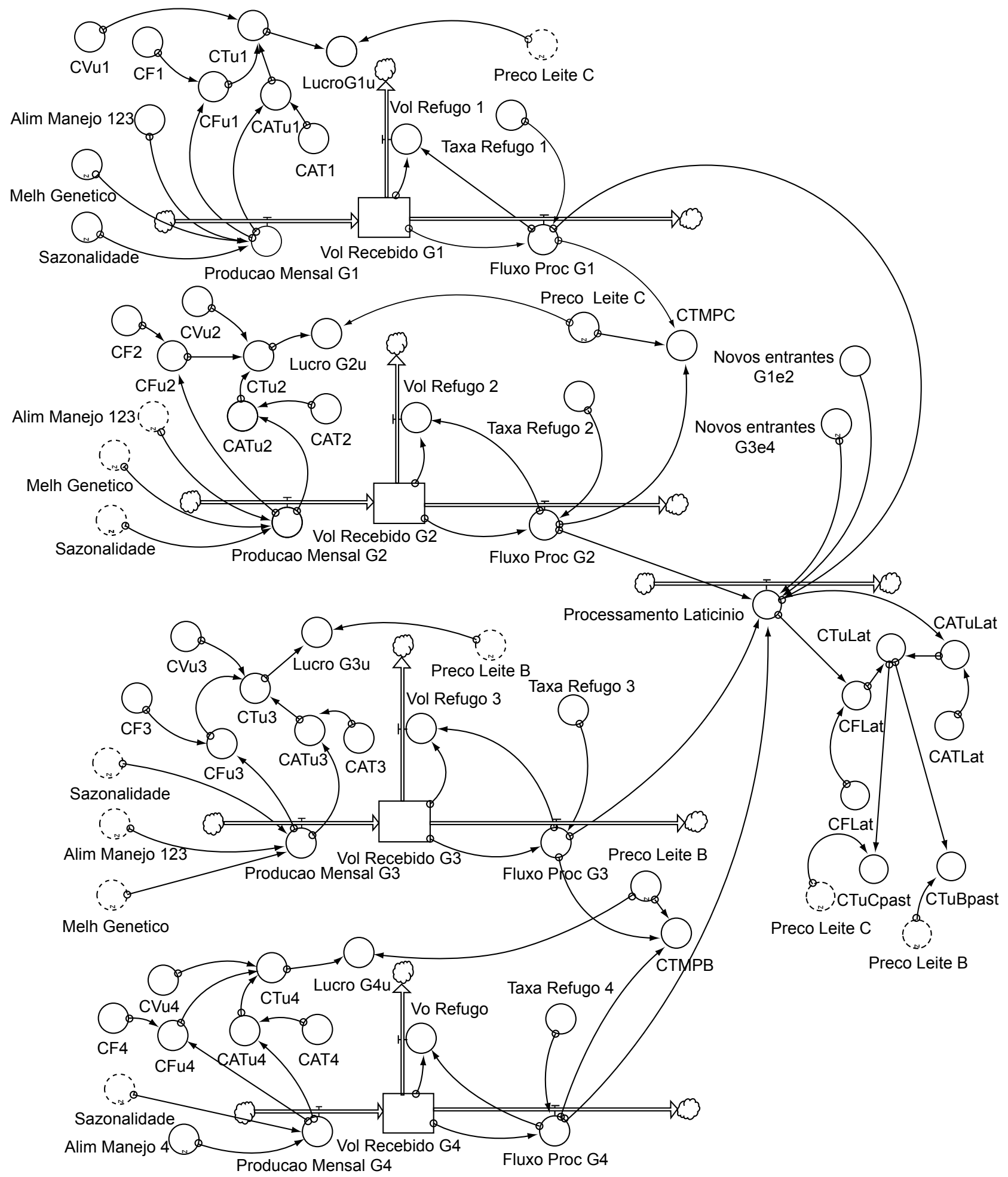

Figura 2. Estrutura do modelo computacional para representação do sistema.

deverá ser respeitada para os demais elementos do modelo. Os estoques são representados pelos elementos "Vol Recebido G1, Vol Recebido G2, Vol Recebido G3 e Vol Recebido G4".

Os fluxos de suprimento foram estabelecidos em se- parado pelos grupos de produtores rurais da rede. Cada grupo é representado pelos produtores selecionados, porém analisados de maneira conjunta (todos os produtores somados nos respectivos grupos - os fluxos "Produção Mensal G1, Produção Mensal G2, Produção Mensal G3 
e Produção Mensal G4"). Por sua vez, os fluxos de processamento da usina representam o volume de produção mensal de cada grupo de produtores, descontadas as quantidades de produto recusadas e refugadas na usina (os fluxos "Vol Refugo 1, Vol Refugo 2, Vol Refugo 3 e Vol Refugo 4"). Deste modo, o fluxo de processamento total ("Processamento Laticínio") é a soma dos fluxos "líquidos" (descontadas as taxas de refugos médias apuradas para os grupos - "Taxa Refugo 1, Taxa Refugo 2, Taxa Refugo 3 e Taxa Refugo 4") dos quatro grupos de produtores de leite, os quais são representados pelos elementos de modelagem "Fluxo Proc G1, Fluxo Proc G2, Fluxo Proc G3 e Fluxo Proc G4".

Os fluxos indicados no modelo apresentam como valor inicial o mês de novembro de 2002. Portanto, a simulação inicia-se por este mês (mês 0 do sistema computacional). O horizonte de tempo determinado para o projeto foi o de 5 anos (60 meses), sendo que todas as variáveis e elementos do modelo devem ser definidas no modelo e ter seu valor calculado para todo este horizonte de tempo.

Buscando representar as variáveis que potencialmente agem sobre o desempenho operacional das propriedades rurais, alguns fatores são estabelecidos no modelo, por meio dos elementos de modelagem variáveis auxiliares (ou conversores). Fatores como a sazonalidade da produção leiteira são descritos de acordo com o período de tempo adequado, por meio da variável "Sazonalidade". Este fator influencia a produção de leite nas fazendas e, conseqüentemente, influencia a utilização de capacidade tanto de produtores rurais quanto da Cooperativa.

Outras duas variáveis representadas no modelo são as variáveis "Alim Manejo 123" e "Alim Manejo 4". A primeira representa o ganho potencial (porcentual) obtido na produção de leite pelo manejo assistido tecnicamente, para os grupos de produtores mais "carentes" de assistência técnica (grupos 1, 2 e 3). A segunda representa o ganho potencial para o grupo de produtores que já utiliza técnicas mais adequadas de manejo e alimentação do gado leiteiro (grupo 4 de produtores). Projeta-se também a influência das ações de melhoramento genético ("Melh Genetico") na produção de leite ao longo do tempo, traduzida em aumento porcentual da capacidade leiteira por animal, no sistema de produção das fazendas.

Cabe destacar que todos esses índices foram devidamente estudados e projetados em conjunto com o corpo técnico da Cooperativa. Especificamente, a influência da variável "Alim Manejo 123" foi baseada também em estudos realizados por Novaes et al. (2001). Neste estudo, os autores estudaram, durante três anos, seis estabelecimentos rurais do interior do Estado de São Paulo (mesma região da Cooperativa em questão), nos quais foram implantados ações agrícolas (uso intensivo de pastagens para o período de chuvas, cana-de-açúcar mais uréia, como suplemento alimentar na seca, e controle reprodutivo e sanitário dos animais), ações ambientais (recuperação e conservação da fertilidade do solo, plantio de matas ciliares, controle de efluentes e melhoria da qualidade da água) e alguns aspectos gerenciais (embora não citados em detalhes). A Figura 3 apresenta o comportamento projetado para a influência da sazonalidade, o potencial de ganho operacional de manejo para os grupos 1,2 e 3 e para o grupo 4 de produtores rurais da rede (variáveis de simulação do modelo).

Na Figura 3, apresenta-se também a influência das ações de melhoramento genético ("Melh Genetico") na produção de leite ao longo do tempo, traduzida em aumento porcentual da capacidade leiteira por animal, no sistema de produção das fazendas. O projeto da influência desta variável evidencia uma importante defasagem técnica entre o início das ações e os seus respectivos impactos na produção de leite das fazendas (o modelo representa potencial de influência apenas para os produtores dos grupos 1, 2 e 3 ).

Esta variável que representa as ações de assistência técnica, para o melhoramento genético dos animais da rede de produtores ("Melh Genetico") tem um comportamento projetado compatível com sua intrínseca defasagem temporal. Para que os ganhos advindos do melhoramento genético sejam obtidos, são necessários considerar os tempos de gravidez dos animais da rede e o tempo de crescimento e maturação, para início da vida útil de lactação dos animais melhorados geneticamente para a atividade leiteira. Considera-se um ciclo constante de novos lactantes e animais de descarte (equilíbrio de número de animais em produção). É importante destacar que não está previsto, em nenhuma das variáveis descritas, o aumento do número de animais nas propriedades da rede. Apenas ganhos de operação e de produtividade animal são previstos neste estudo de simulação. O resu ltado da simulação pode ser avaliado pela construção das variáveis de custo operacional dos agentes. Os custos variáveis unitários ("CVu1, Cvu2, Cvu3 e Cvu4") e custos fixos totais mensais médios ("CF1, CF2, CF3 e CF4") dos grupos produtores rurais foram obtidos na pesquisa de campo. Custos adicionais após o início e aceitação do projeto, para os produtores rurais, também foram previstos pelo modelo ("CAT1, CAT2, CAT3 e CAT4"). Esses custos adicionais representam um aumento no custo mensal, com alimentação e investimentos nas instalações de manejo dos produtores, da ordem de $10 \%$ do faturamento médio mensal dos produtores dos grupos considerados. As equações e os dados de entrada do modelo estão apresentados no Anexo I.

Os custos fixos unitários ("CFu1, CFu2, CFu3 e CFu4") e custos adicionais unitários ("CATu1, CATu2, CATu3 e CATu4") são calculados pelo sistema a cada "rodada" do 
modelo no horizonte de tempo do projeto. Da diferença dos custos unitários totais de cada grupo ("CTu1, CTu2, CTu3 e CTu4") e dos preços pagos para os leites C e B projetados pela Cooperativa, pode-se prever o resultado operacional unitário de cada grupo de produtores rurais, os quais estão representados no modelo pelas variáveis "Lucro G1u”, "Lucro G2u”, "Lucro G3u" e "Lucro G4u”. Estas variáveis do modelo (na verdade representam um lucro ou mesmo um prejuízo operacional) são resultados importantes do modelo de simulação.

Cabe destacar que o fator identificado nas entrevistas de campo, como sendo crucial para o sistema, foi o estabelecimento de políticas mais adequadas de preços para a atividade pecuária, além do estabelecimento de ações de assistência técnica. Nesse sentido, projetou-se uma elevação dos preços pagos ao produtor de leite $\mathrm{C} \mathrm{em} 20 \%$, para o $13^{\circ}$ mês do projeto (primeira estação de maior oferta após o início da simulação), passando de $\mathrm{R} \$ 0,38$ por litro (preço vigente quando da realização do trabalho) para
$\mathrm{R} \$ 0,45$. Além disso, projetou-se também um aumento adicional de $12 \%$ para os dois últimos períodos de safra ( $49^{\circ}$ e $60^{\circ}$ mês de simulação), passando de $\mathrm{R} \$ 0,45$ para $\mathrm{R} \$ 0,50$. Cabe destacar que os preços projetados são, a partir do segundo mês de simulação, $10 \%$ maiores que o preço praticado pelo mercado regional. Em contrapartida, poder-se-ia esperar uma mudança de comportamento do produtor traduzida pela busca por maiores índices de produtividade, para aproveitar os mais altos preços, mesmo que estes devam arcar com os maiores custos com alimentação de melhor rendimento operacional para $o$ gado leiteiro. Para o leite Tipo B, os porcentuais e as datas programadas para os incrementos nos preços foram os mesmos estabelecidos para o leite Tipo C.

Os resultados da simulação evidenciam a existência de ganhos potenciais para os quatro grupos de produtores rurais (Figura 4). Estes resultados mostram uma situação de dificuldades operacionais para os grupos 1,2 e 3 do modelo, a começar pela situação inicial destes, calculada

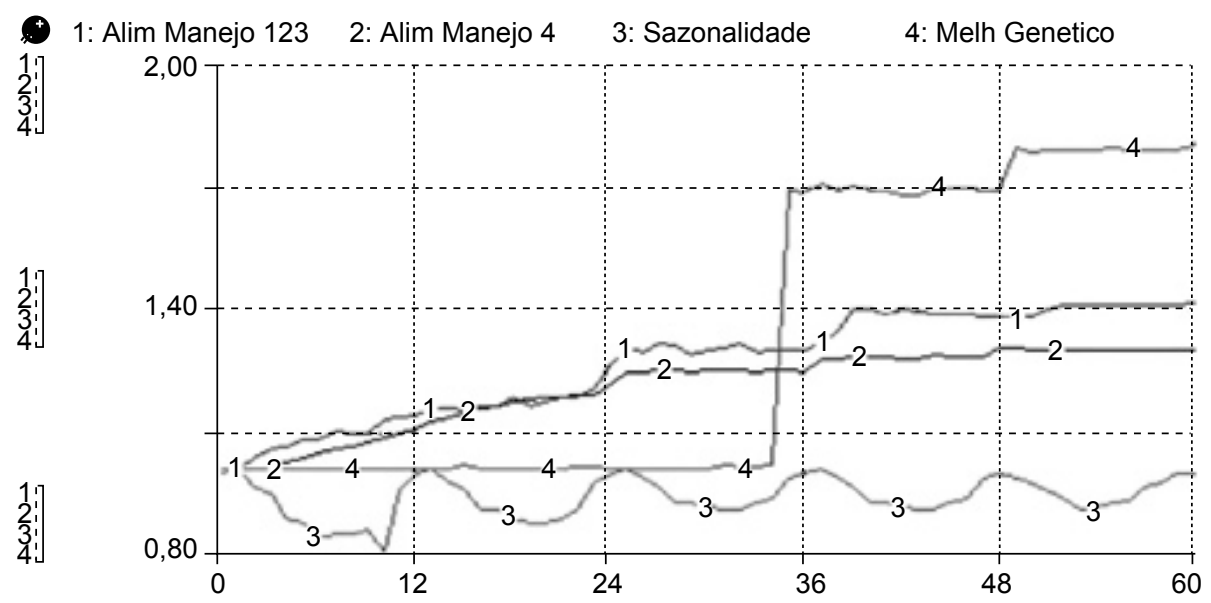

Figura 3. Variáveis de simulação: Comportamento projetado para a influência das ações de assistência técnica, melhoramento genético e da sazonalidade na produção rural.

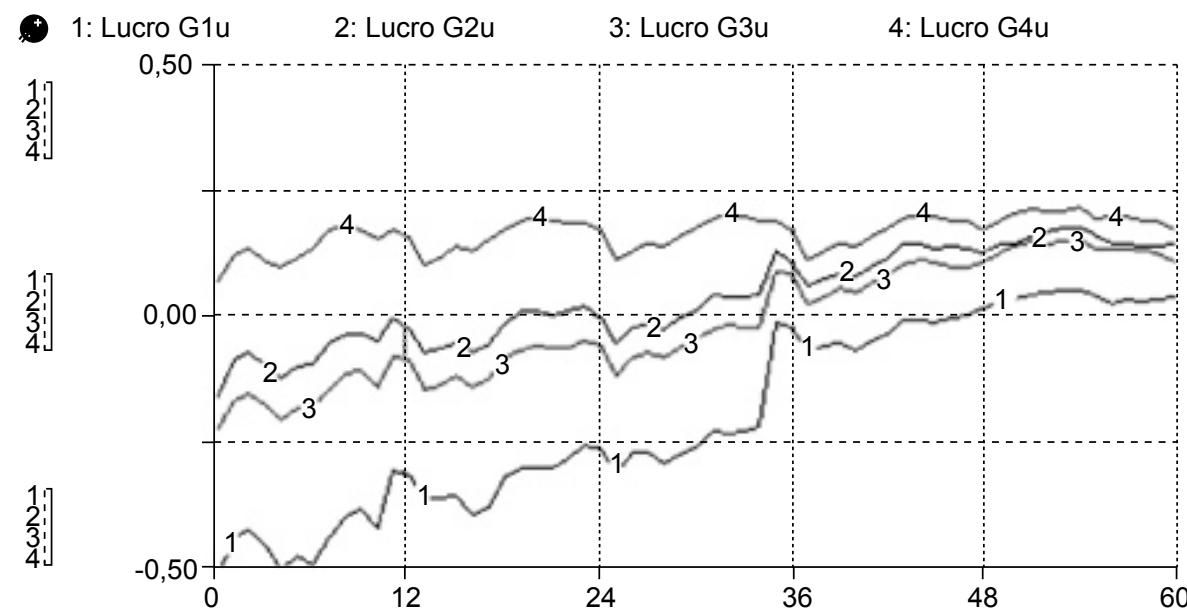

Figura 4. Rentabilidade operacional para os quatro grupos de produtores rurais do modelo. 
pelo modelo e, pelo menos, durante os primeiros três anos do projeto. A partir do final do terceiro ano de simulação, evidencia-se um importante ponto de alavancagem para os produtores do sistema. Este ponto do projeto é o ponto no qual há a efetiva renovação (em qualidade e capacidade de lactação) dos animais da rede, fruto do programa de melhoramento genético. Esses resultados mostram que há alternativas para a questão da viabilidade econômica das pequenas propriedades rurais, no contexto do agronegócio nacional, desde que estas sejam inseridas em projetos de reestruturação produtiva.

Pode-se esperar também que os custos operacionais de processamento industrial (laticínio) sejam reduzidos. No sentido de avaliar esta possibilidade, nesta simulação econômica, para avaliação de alternativas, foi proposto que fosse de responsabilidade da Cooperativa a realização de tarefas como a análise de pastagens, controle de zoonoses mais efetivo, melhoria genética de animais, etc. Os custos mensais com pessoal especializado e materiais diversos de acompanhamento médico dos animais e de inseminação artificial, para melhoramento genético dos animais da rede, foram, deste modo, triplicados. Este custo foi baseado em padrões estabelecidos em situações já experimentadas pela Cooperativa no passado.

Outra variável importante prevista no modelo é a possibilidade de novos entrantes na rede, fruto do reconhecimento, por parte dos produtores rurais da região da Cooperativa, das novas políticas de preços e de assistência técnica. As variáveis "Novos Entrantes G1e2" e "Novos Entrantes G3e4" podem representar a possibilidade tanto de perda quanto de recuperação de produtores dos grupos 1 e 2 e dos grupos 3 e 4 , respectivamente (são, portanto, variáveis de simulação).

Do lado do processamento industrial (Cooperativa), espera-se também ganhos operacionais. Os custos das usinas processadoras foram tratados de maneira similar. O custo fixo total da Cooperativa foi representado pela variável "CFLat" no modelo. Por sua vez, o custo de assistência técnica (fixo também) foi representado pela variável "CATLat". Os respectivos custos unitários são calculados a cada "rodada" do sistema e são obtidos da divisão dos valores totais pelo processamento mensal de leite (Tipos B e C somados).

Cabe destacar que o custo de processamento dos leites Tipo B e C (variável auxiliar do modelo "CTuLat") refere-se à maior parte dos custos fixos indiretos ao processo produtivo (exceto os custos comerciais), os quais são ocasionados pela operação básica da Cooperativa: a recepção e a pasteurização dos leites C e B. Estes custos incidem, quaisquer que sejam suas finalidades comerciais posteriores. Por exemplo, tanto para fabricação de queijo (fábrica de queijos é um setor específico dentro da usina central) ou mesmo dos leites pasteurizados C e B envasados em caixas ou sacos plásticos (caso da usina), a matéria-prima (leite cru) necessita ser pasteurizada e posteriormente destinada a processos de produção, em função de sua finalidade comercial. Geram-se, a partir deste ponto, novos custos operacionais. Esses novos custos são custos específicos a cada nova operação do processo produtivo e são adicionados ao custo de processamento, para formar o custo final das linhas de produtos.

Atividades de laboratório, recepção, custos das atividades de suporte de geração de vapor (caldeiras), tratamento de efluentes, sistema de refrigeração, gerência de produção, gerência de produtores e parte significativa dos custos administrativos foram devidamente alocados ao custo fixo do processo citado.

$\mathrm{O}$ custo de processamento unitário, adicionado ao custo de aquisição do leite cru Tipo $\mathrm{C}$ e $\mathrm{B}$, forma os custos completos dos leites $\mathrm{C}$ e $\mathrm{B}$ processados pela empresa ("CTuCpast e CTuBpast"). A Figura 5 mostra o resultado da simulação para estes custos. Esperava-se que esses custos tivessem um comportamento de queda, com o aumento da utilização da capacidade e maior regularidade de suprimento. De fato, os resultados mostram este comportamento esperado, conferindo ao sistema uma resposta positiva em relação aos aspectos de viabilidade operacional do projeto em questão.

Os custos são fortemente influenciados pelo fator sazonal e apresentam um valor inicial bastante elevado para os padrões de preço final, praticado para os produtos lácteos oferecidos pela empresa. Porém, o fator sazonal é projetado para ter sua influência reduzida ao longo do horizonte do projeto, por conta das ações de intervenção e de apoio técnico propostas.

Entretanto, a rentabilidade operacional do laticínio dependerá da comparação entre os custos operacionais e sua performance de mercado. Por sua vez, os custos operacionais dependem fortemente da escala de produção, a qual é fortemente dependente da performance rural da rede. Esta análise complementar configura-se numa extensão deste trabalho.

\section{Considerações finais}

Acredita-se que o método proposto neste trabalho seja um ferramental alternativo para auxiliar nos processos de reestruturação organizacional e operacional, em cadeias de suprimento empresariais.

Propõe-se que a mesma seja útil no sentido de auxiliar os tomadores de decisão a encontrarem um caminho para medir e reportar custos e outras informações gerenciais, além das fronteiras da firmas individuais, compartilhando informação sobre o nível dos benefícios e com quais membros eles estão (ou estarão). Além disso, pode-se evidenciar (e evitar) situações que possam colocar alguns 


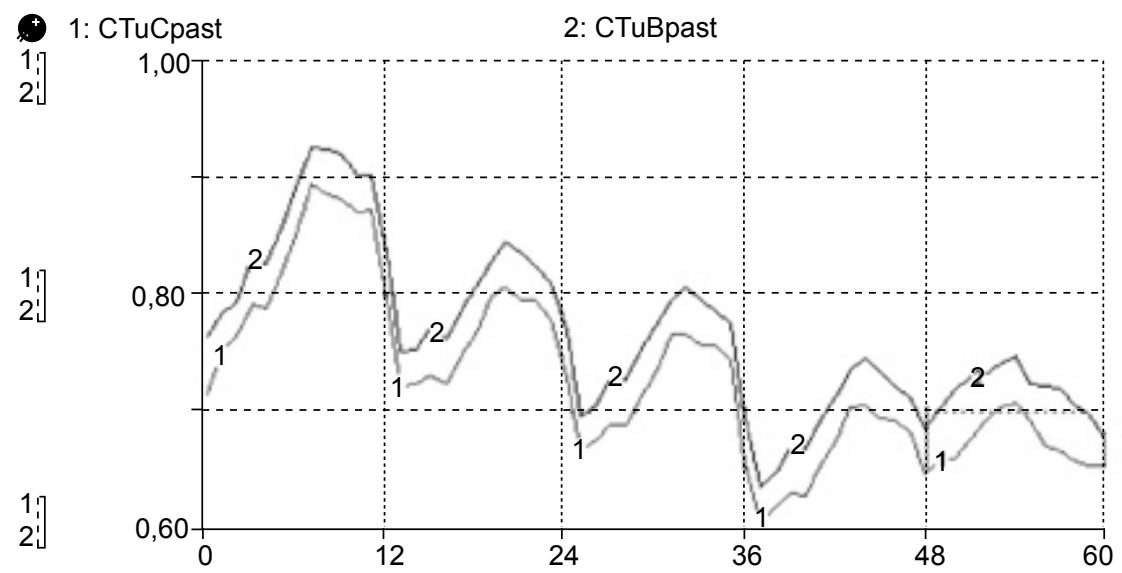

Figura 5. Resultado de simulação para os custos dos leites pasteurizados C e B (antes do processamento final das linhas de comercialização).

membros da cadeia de suprimento em processo de reestruturação em situações piores às anteriores, como resultado de sua cooperação.

Todavia, é importante que o ferramental computacional (o modelo) seja utilizado como um mecanismo de retroalimentação (feedback), para verificar a eficácia das projeções das variáveis de simulação e dos resultados operacionais esperados para os agentes. Evidentemente os resultados dependem das considerações e aproxima- ções realizadas para as variáveis de simulação.

Entretanto, destas considerações pode-se dizer que, uma vez aplicado o método, uma das grandes vantagens torna-se a possibilidade de testar especificações alternativas para o funcionamento do sistema. Assim, com grande agilidade e rapidez, um amplo campo de modelos e de cenários alternativos pode ser testado, apenas variando o comportamento e as especificações das variáveis do modelo.

\section{Referências Bibliográficas}

BALLOU, R. H.; GILBERT, S. M.; MUKHERJEE, A. New managerial challenges from supply chain opportunities. Industrial Marketing Management, v. 29, p. 7-18, 2000.

BATALHA, M. O.; SILVA, A. L. Gestão de cadeias produtivas: novos aportes teóricos e empíricos. São Carlos, 1999 (mimeo). Documento preliminar para discussão Departamento de Engenharia de Produção da UFSCar.

BUONGIORNO, J. Forest sector modeling: a syntesis of econometrics, mathematical programming, and a system dynamics methods. International Journal of Forecasting, v. 12, p. 329-343, 1996.

COOPER, M. C.; LAMBERT, D. M.; PAGH, J. D. Supply chain management: more than a new name for logistics. The International Journal of Logistics Management, v. 8, n. 1, p. 1-13, 1997.

COVER, J. Introduction to system dynamics. 1. ed. Reston: Powersim Press, 1996. 82 p.

FISHER, D. K.; NORVELL, J.; SONKA, S.; NELSON, M. J. Understanding technology adoption through system dynamics modeling: implications for agribusiness. Management International Food and Agribusiness Management Review, v. 3, p. 281-296, 2000.

GILBERT, S. M.; BALLOU, R. H. Supply chain benefits from advanced customer commitments. Journal of Operations Management, v.18, p. 61-73, 1999.

NOVAES, N. J.; CAMARGO, A. C.; MANZANO, A.; ESTEVES, S. N.; FREITAS, A. R. de; TUPY, O.; MACHADO, R. Avaliação de Tecnologias Agropecuárias em Estabelecimentos Familiares com Produção de Leite no Município de São Carlos, SP. In: CONGRESSO DE AGROPECUÁRIA DE LEITE, 11., 2001, viçosa - MG. Anais....Viçosa, 2001. CD-ROM.

SAITO, J. R., Análise de cadeias agroindustriais utilizando simulação computacional baseada na metodologia de system dynamics: um estudo de caso. 2000. Dissertação (Mestrado em Engenharia de Produção) - Departamento de Engenharia de Produção, Universidade Federal de São Carlos, São Carlos, 2000.

SMITH, P. C.; VAN ACKERE, A. A note on the integration of system dynamics and economic models. Journal of Economic Dynamics \& Control, v. 26, p. 1-10, 2002.

SONKA, S. T.; CLOUTIER, L. M. System dynamics to evaluate information coordination in agricultural supply chains. Revista Brasileira de Agroinformática, v. 1, n. 1, p. 1-16, 1998.

STELLA and STELLA Research: an introduction to system thinking. Hanover: High Performance Systems, 
Inc., 1997. 196 p.

ZIMMER, K. Supply chain coordination with uncertain just-in-time delivery. International Journal of Production Economics, v. 77, n. 1, p. 1-15, 2002.
YONENAGA, W. H., Utilização de System Dynamics no gerenciamento de florestas plantadas. 2001. Dissertação (Mestrado em Engenharia de Produção) - Departamento de Engenharia de Produção, Universidade Federal de São Carlos, São Carlos, 2001.

\title{
A METHOD TO ANALYZE BENEFITS IN SUPPLY CHAIN BENEFITS: A CASE STUDY IN THE BRAZILIAN DAIRY SECTOR
}

\begin{abstract}
This paper proposes an analytical method, based on a framework that integrates the concepts of Supply Chain Management and Cost Management Systems, to study and restructure Brazilian agricultural supply chains. Using systemic reasoning and the system dynamics $(S D)$ approach, the proposed method was applied to Brazil's dairy sector to quantitatively analyze the performance of organizational and technical configurations of the sector's economic agents. A network of companies in Brazil's dairy supply chain was defined in terms of an SD model, which indicated that the impact of actions on the agents' production costs can be forecasted before such actions are taken, conferring greater consistency on the study. The network consisted of four representative groups of rural producers and a dairy company. Input data were based on a network operating in the central region of the state of Sao Paulo. The proposed method also proved highly applicable as a management tool in the reformulation of public and/or private policies aimed at enhancing the efficiency of economic and productive systems (agricultural supply chains).
\end{abstract}

Keywords: supply chain management, systemic reasoning, computational modeling.

\section{Anexo I: Especificações do modelo (equações e dados de entrada)}

Vol_Recebido_G1 $(\mathrm{t})=$ Vol_Recebido_G1 $(\mathrm{t}-\mathrm{dt})+$ (Producao_Mensal_G1 - Flxuo_Proc_G1 - Vol_Refugo1) $* \mathrm{dt}$

INIT Vol_Recebido_G1 $=112 * 59.76 * 30$

INFLOWS:

Producao_Mensal_G1 $=112 * 59.76 * 30 *$ AlimManejo

$123 *$ MelhGenetico*Sazonalidade

OUTFLOWS:

Flxuo_Proc_G1 = Vol_Recebido_G1*(1-TaxaRefugo1)

Vol_Refugo1 $=$ Vol_Recebido_G1-Flxuo_Proc_G1

Vol_Recebido_G2 $(\mathrm{t})=$ Vol_Recebido_G2 $(\mathrm{t}-\mathrm{dt})+$ (Producao_Mensal_G2 - Vol_Refugo_2 - Fluxo_Proc_ $\mathrm{G} 2) * \mathrm{dt}$

INIT Vol_Recebido_G2 $=26 * 258.7 * 30$

INFLOWS:

Producao_Mensal_G2 $=26 * 258.7 * 30 *$ AlimManejo1 $23 *$ MelhGenetico*Sazonalidade

OUTFLOWS:

Vol_Refugo_2 = Vol_Recebido_G2-Fluxo_Proc_G2 Fluxo_Proc_G2 = Vol_Recebido_G2*(1-TaxaRefugo2)
Vol_Recebido_G3(t) $=$ Vol_Recebido_G3(t $-\mathrm{dt})+$ (Producao_Mensal_G3 - Fluxo_Proc_G3 - Vol_Refugo_3) $* \mathrm{dt}$

INIT Vol_Recebido_G3 $=5 * 197.67 * 30$

INFLOWS:

Producao_Mensal_G3 $=5 * 197.67 * 30 *$ Sazonalidade*

MelhGenetico*AlimManejo123

OUTFLOWS:

Fluxo_Proc_G3 = Vol_Recebido_G3*(1-TaxaRefugo3)

Vol_Refugo_3 =Vol_Recebido_G3-Fluxo_Proc_G3

Vol_Recebido_G4 $(\mathrm{t})=$ Vol_Recebido_G4 $(\mathrm{t}-\mathrm{dt})+$ (Producao_Mensal_G4 - Vol_Refugo_4 - Fluxo_Proc_ G4) *dt

INIT Vol_Recebido_G4 $=5 * 1212.7 * 30$

INFLOWS:

Producao_Mensal_G4 $=5 * 1212.7 * 30 *$ AlimManejo4 *Sazonalidade

OUTFLOWS:

Vol_Refugo_4 = Vol_Recebido_G4-Fluxo_Proc_G4

Fluxo_Proc_G4 = Vol_Recebido_G4 $*(1-$ TaxaRefugo4)

CAT1 $=\operatorname{DELAY}(112 * 68.13,3,0)$ 
CAT2 $=$ DELAY $(26 * 294.92,3,0)$

CAT3 $=\operatorname{DELAY}(5 * 254.99,3,0)$

CAT4 $=$ DELAY $(5 * 1563.48,3,0)$

CATLat $=$ DELAY $(22500,3,8430)$

CATu $1=$ CAT1/Producao_Mensal_G1

CATu2 $=$ CAT2/Producao_Mensal_G2

CATu3 $=$ CAT3/Producao_Mensal_G3

CATu4 $=$ CAT4/Producao_Mensal_G4

CATuLat $=$ CATLat $/$ Processamento_Laticínio

$\mathrm{CF} 1=112 * 1196.91$

$\mathrm{CF} 2=26 * 2198.19$

$\mathrm{CF} 3=5 * 2162.81$

$\mathrm{CF} 4=5 * 5181.58$

CFbEmp $=(49101.91-13101.82) * 0.5$

$\mathrm{CFbEmpU}=\mathrm{CFbEmp} /$ Leite_B

$\mathrm{CFcEmp}=(49101.91-13101.82) * 0.5$

$\mathrm{CFcEmpU}=\mathrm{CFcEmp} /$ Leite_C

CFLat $=38185.09+13707.61+92208+59004.55$

CFu1 $=$ CF1/Producao_Mensal_G1

CFu2 $=$ CF2/Producao_Mensal_G2

CFu3 $=$ CF3/Producao_Mensal_G3

CFu4 = CF4/Producao_Mensal_G4

CFuLAt $=$ CFLat/Processamento_Laticínio

CTEmbDist $=(\text { Leite_B+Leite_C })^{*}$ CVuEmbDist

CTMPB $=(\text { Fluxo_Proc_G3+Fluxo_Proc_G4 })^{*}$ Preco_

Leite_B

CTMPC $=($ Flxuo_Proc_G1+Fluxo_Proc_G2 $) *$ Preco_

Leite_C

$\mathrm{CTu} 1=\mathrm{CFu} 1+\mathrm{CVu} 1+\mathrm{CATu} 1$

$\mathrm{CTu} 2=\mathrm{CFu} 2+\mathrm{CVu} 2+\mathrm{CATu} 2$

$\mathrm{CTu} 3=\mathrm{CFu} 3+\mathrm{CVu} 3+\mathrm{CATu} 3$

$\mathrm{CTu} 4=\mathrm{CFu} 4+\mathrm{CVu} 4+\mathrm{CATu} 4$

CTuBpast $=$ CTuLat+Preco_Leite_B

CTuCpast $=$ Preco_Leite_C+CTuLat

CTuLat $=$ CATuLat + CFuLAt

$\mathrm{CVu} 1=0.23$

$\mathrm{CVu} 2=0.26$

$\mathrm{CVu} 3=0.29$

$\mathrm{CVu} 4=0.22$

LucroG1u $=$ Preco_Leite_C-CTu1

LucroG2 $\mathrm{u}=$ Preco_Leite_C-CTu2

LucroG3u $=$ Preco_Leite_B-CTu3

LucroG4u $=$ Preco_Leite_B-CTu4

TaxaRefugo $1=0$

TaxaRefugo $2=0$

TaxaRefugo3 $=0$

TaxaRefugo $4=0$

AlimManejo123 = GRAPH(TIME)

$(0,00,1,00) ;(1,00,1,00) ;(2,00,1,03) ;(3,00,1,05)$; $(4,00,1,06) ;(5,00,1,08) ;(6,00,1,08) ;(7,00,1,10) ;(8,00$, 1,09); (9,00, 1,09); (10,0, 1,13); (11,0, 1,13); (12,0, 1,14); $(13,0,1,15) ;(14,0,1,15) ;(15,0,1,15) ;(16,0,1,16) ;(17,0$, $1,16) ;(18,0,1,18) ;(19,0,1,16) ;(20,0,1,17) ;(21,0,1,18)$;
$(22,0,1,18) ;(23,0,1,20) ;(24,0,1,27) ;(25,0,1,30) ;(26,0$, $1,29) ;(27,0,1,31) ;(28,0,1,31) ;(29,0,1,29) ;(30,0,1,30)$; $(31,0,1,30) ;(32,0,1,31) ;(33,0,1,29) ;(34,0,1,30) ;(35,0$, $1,30) ;(36,0,1,30) ;(37,0,1,32) ;(38,0,1,35) ;(39,0,1,40)$; $(40,0,1,40) ;(41,0,1,39) ;(42,0,1,40) ;(43,0,1,39) ;(44,0$, 1,39); (45,0, 1,39); (46,0, 1,39); (47,0, 1,38); (48,0, 1,38); $(49,0,1,38) ;(50,0,1,38) ;(51,0,1,40) ;(52,0,1,41) ;(53,0$, $1,41) ;(54,0,1,41) ;(55,0,1,41) ;(56,0,1,41) ;(57,0,1,41)$; $(58,0,1,41) ;(59,0,1,41) ;(60,0,1,41)$

AlimManejo4 = GRAPH(TIME)

$(0,00,1,00) ;(1,00,1,00) ;(2,00,1,00) ;(3,00,1,00)$; $(4,00,1,02) ;(5,00,1,03) ;(6,00,1,04) ;(7,00,1,05) ;(8,00$, $1,06) ;(9,00,1,07) ;(10,0,1,08) ;(11,0,1,09) ;(12,0,1,10)$; $(13,0,1,12) ;(14,0,1,13) ;(15,0,1,14) ;(16,0,1,15) ;(17,0$, $1,16) ;(18,0,1,17) ;(19,0,1,18) ;(20,0,1,18) ;(21,0,1,18)$; $(22,0,1,18) ;(23,0,1,19) ;(24,0,1,21) ;(25,0,1,24) ;(26,0$, $1,24) ;(27,0,1,25) ;(28,0,1,25) ;(29,0,1,24) ;(30,0,1,25)$; $(31,0,1,25) ;(32,0,1,25) ;(33,0,1,24) ;(34,0,1,25) ;(35,0$, $1,25) ;(36,0,1,24) ;(37,0,1,27) ;(38,0,1,27) ;(39,0,1,28)$; $(40,0,1,28) ;(41,0,1,28) ;(42,0,1,28) ;(43,0,1,28) ;(44,0$, $1,28) ;(45,0,1,28) ;(46,0,1,28) ;(47,0,1,28) ;(48,0,1,30)$; $(49,0,1,30) ;(50,0,1,30) ;(51,0,1,30) ;(52,0,1,30) ;(53,0$, $1,30) ;(54,0,1,30) ;(55,0,1,30) ;(56,0,1,30) ;(57,0,1,30)$; $(58,0,1,30) ;(59,0,1,30) ;(60,0,1,30)$

MelhGenetico $=$ GRAPH $($ TIME $)$

$(0,00,1,00) ;(1,00,1,00) ;(2,00,1,00) ;(3,00,1,00)$; $(4,00,1,00) ;(5,00,1,00) ;(6,00,1,00) ;(7,00,1,00) ;(8,00$, $1,01) ;(9,00,1,01) ;(10,0,1,01) ;(11,0,1,01) ;(12,0,1,01)$; $(13,0,1,00) ;(14,0,1,00) ;(15,0,1,02) ;(16,0,1,01) ;(17,0$, $1,01) ;(18,0,1,01) ;(19,0,1,01) ;(20,0,1,01) ;(21,0,1,01)$; $(22,0,1,01) ;(23,0,1,01) ;(24,0,1,01) ;(25,0,1,01) ;(26,0$, $1,01) ;(27,0,1,01) ;(28,0,1,01) ;(29,0,1,01) ;(30,0,1,01)$; $(31,0,1,02) ;(32,0,1,01) ;(33,0,1,01) ;(34,0,1,02) ;(35,0$, $1,69)$; $(36,0,1,69) ;(37,0,1,71) ;(38,0,1,69) ;(39,0,1,70)$; (40,0, 1,69); (41,0, 1,69); (42,0, 1,68); (43,0, 1,68); (44,0, $1,70) ;(45,0,1,70) ;(46,0,1,70) ;(47,0,1,69) ;(48,0,1,69)$; $(49,0,1,80) ;(50,0,1,79) ;(51,0,1,79) ;(52,0,1,79) ;(53,0$, $1,79) ;(54,0,1,79) ;(55,0,1,80) ;(56,0,1,79) ;(57,0,1,79)$; $(58,0,1,79) ;(59,0,1,79) ;(60,0,1,80)$

Novos_entrantes_G1e2 $=$ GRAPH(TIME)

$(0,00,0,00) ;(1,03,80256) ;(2,07,83493) ;(3,10$, 99333); (4,14, 113466); (5,17, 119624); $(6,21,82261)$; $(7,24,84493) ;(8,28,90447) ;(9,31,68132) ;(10,3$, 96461); (11,4, 79737); (12,4, 86943); (13,4, 149864); (14,5, 184733); (15,5, 212624); (16,6, 219927); (17,6, 234428); (18,6, 233149); (19,7, 219146); (20,7, 218837); (21,7, 199746); (22,8, 208779); (23,8, 197243); (24,8, 213077); (25,9, 250245); (26,9, 247750); (27,9, 258892); (29,0, 279122); (30,0, 275345); (31,0, 265084); (32,1, 267672); (33,1, 260968); (34,1, 248239); (35,2, 251060); (36,2, 248549); (37,2, 257400); (38,3, 277098); (39,3, 265873); (40,3, 274567); (41,4, 275966); (42,4, 281293); (43,4, 282500); (44,5, 282500); (45,5, 282500); (46,6, 
282500); (47,6, 287500); (48,6, 290000); (49,7, 290000); (50,7, 287500); (51,7, 287500); (52,8, 287500); (53,8, 287500); (54,8, 287500); (55,9, 287500); (56,9, 287500); $(57,9,287500) ;(59,0,287500) ;(60,0,287500)$

Novos_Entrantes_G3e4 = GRAPH(TIME)

$(0,00,24202) ;(1,00,16138) ;(2,00,27000) ;(3,00$, $30000) ;(4,00,31000) ;(5,00,29000) ;(6,00,30000)$; $(7,00,30000) ;(8,00,30000) ;(9,00,30000) ;(10,0$, 30000); (11,0, 30000); (12,0, 28000); (13,0, 29000); $(14,0,29000) ;(15,0,29000) ;(16,0,49395) ;(17,0$, 47187); (18,0, 48000); (19,0, 47000); (20,0, 47000); $(21,0,47000) ;(22,0,50000) ;(23,0,50000) ;(24,0$, 75000); (25,0, 75000); (26,0, 75000); (27,0, 75000); $(28,0,75000) ;(29,0,74000) ;(30,0,74000) ;(31,0$, 72000); (32,0, 70000); (33,0, 75000); (34,0, 75000); $(35,0,75000) ; \quad(36,0,75000) ;(37,0,75000) ; \quad(38,0$, 75000); (39,0, 75000); (40,0, 75000); (41,0, 75000); $(42,0,75000) ;(43,0,75000) ;(44,0,75000) ;(45,0$, 75000); (46,0, 75000); (47,0, 75000); (48,0, 75000); $(49,0,75000) ;(50,0,75000) ;(51,0,75000) ; \quad(52,0$, $75000)$; (53,0, 75000); (54,0, 75000); (55,0, 75000); $(56,0,75000) ;(57,0,75000) ;(58,0,75000) ;(59,0$, 75000); (60,0, 75000)

Preco_Leite_B $=$ GRAPH(TIME)

$(0,00,0,43) ;(1,00,0,48) ;(2,00,0,5) ;(3,00,0,52)$; $(4,00,0,52) ;(5,00,0,54) ;(6,00,0,56) ;(7,00,0,58) ;(8,00$, $0,59) ;(9,00,0,58) ;(10,0,0,57) ;(11,0,0,56) ;(12,0,0,54)$; $(13,0,0,48) ;(14,0,0,5) ;(15,0,0,52) ;(16,0,0,52) ;(17,0$, $0,54) ;(18,0,0,56) ;(19,0,0,58) ;(20,0,0,59) ;(21,0,0,58)$; $(22,0,0,57) ;(23,0,0,56) ;(24,0,0,54) ;(25,0,0,48) ;(26,0$, $0,5) ;(27,0,0,52) ;(28,0,0,52) ;(29,0,0,54) ;(30,0,0,56)$; $(31,0,0,58) ;(32,0,0,59) ;(33,0,0,58) ;(34,0,0,57) ;(35,0$, $0,56) ;(36,0,0,54) ;(37,0,0,48) ;(38,0,0,5) ;(39,0,0,52)$; $(40,0,0,52) ;(41,0,0,54) ;(42,0,0,56) ;(43,0,0,58) ;(44,0$,
$0,59) ;(45,0,0,58) ;(46,0,0,57) ;(47,0,0,56) ;(48,0,0,54)$; $(49,0,0,56) ;(50,0,0,578) ;(51,0,0,588) ;(52,0,0,588)$; $(53,0,0,595) ;(54,0,0,6) ;(55,0,0,578) ;(56,0,0,578)$; $(57,0,0,575) ;(58,0,0,565) ;(59,0,0,558) ;(60,0,0,54)$

Preco_Leite_C $=$ GRAPH(TIME)

$(0,00,0,38) ;(1,00,0,45) ;(2,00,0,47) ;(3,00,0,48)$; $(4,00,0,48) ;(5,00,0,5) ;(6,00,0,52) ;(7,00,0,55) ;(8,00$, $0,55) ;(9,00,0,54) ;(10,0,0,54) ;(11,0,0,53) ;(12,0,0,5)$; $(13,0,0,45) ;(14,0,0,47) ;(15,0,0,48) ;(16,0,0,48) ;(17,0$, $0,5) ;(18,0,0,52) ;(19,0,0,55) ;(20,0,0,55) ;(21,0,0,54)$; (22,0, 0,54); (23,0, 0,53); (24,0, 0,5); (25,0, 0,45); (26,0, 0,47); (27,0, 0,48); (28,0, 0,48); (29,0, 0,5); (30,0, 0,52); $(31,0,0,55) ;(32,0,0,55) ;(33,0,0,54) ;(34,0,0,54) ;(35,0$, 0,53); (36,0, 0,5); (37,0, 0,45); (38,0, 0,47); (39,0, 0,48); (40,0, 0,48); (41,0, 0,5); (42,0, 0,52); (43,0, 0,55); (44,0, $0,55) ;(45,0,0,54) ;(46,0,0,54) ;(47,0,0,53) ;(48,0,0,5)$; (49,0, 0,516); (50,0, 0,52); (51,0, 0,534); (52,0, 0,547); $(53,0,0,558) ;(54,0,0,558) ;(55,0,0,545) ;(56,0,0,525)$; $(57,0,0,522) ;(58,0,0,516) ;(59,0,0,513) ;(60,0,0,516)$

Sazonalidade $=$ GRAPH $($ TIME $)$

$(0,00,0,99) ;(1,00,1,00) ;(2,00,0,96) ;(3,00,0,94)$; $(4,00,0,88) ;(5,00,0,87) ;(6,00,0,83) ;(7,00,0,84) ;(8,00$, $0,84) ;(9,00,0,85) ;(10,0,0,8) ;(11,0,0,95) ;(12,0,0,99)$; $(13,0,1,00) ;(14,0,0,97) ;(15,0,0,95) ;(16,0,0,9) ;(17,0$, $0,9) ;(18,0,0,88) ;(19,0,0,87) ;(20,0,0,87) ;(21,0,0,88)$; $(22,0,0,9) ;(23,0,0,97) ;(24,0,0,99) ;(25,0,1,00) ;(26,0$, $0,98) ;(27,0,0,96) ;(28,0,0,92) ;(29,0,0,92) ;(30,0,0,91)$; $(31,0,0,9)$; $(32,0,0,9)$; $(33,0,0,92) ;(34,0,0,93)$; $(35,0$, 0,98); (36,0, 0,99); (37,0, 1,00); (38,0, 0,98); (39,0, 0,96); (40,0, 0,92); (41,0, 0,92); (42,0, 0,91); (43,0, 0,9); (44,0, $0,9) ;(45,0,0,92) ;(46,0,0,93)$; $(47,0,0,98) ;(48,0,0,99)$; $(49,0,0,98) ;(50,0,0,969) ;(51,0,0,949) ;(52,0,0,929)$; $(53,0,0,9) ;(54,0,0,91) ;(55,0,0,922) ;(56,0,0,924)$; $(57,0,0,959) ;(58,0,0,969) ;(59,0,0,989) ;(60,0,0,99)$. 